Philosophie ANTIQUE

\section{Philosophie antique}

Problèmes, Renaissances, Usages

$11 \mid 2011$

Influences, filiations, réceptions (XVIIe-XXe siècles)

\title{
La question du platonisme de Rousseau
}

\section{Francesco Gregorio}

\section{OpenEdition}

\section{Journals}

Édition électronique

URL : http://journals.openedition.org/philosant/993

DOI : 10.4000/philosant.993

ISSN : 2648-2789

\section{Éditeur}

Éditions Vrin

\section{Édition imprimée}

Date de publication : 1 novembre 2011

Pagination : $43-71$

ISBN : 978-2-7574-0356-3

ISSN : $1634-4561$

\section{Référence électronique}

Francesco Gregorio, «La question du platonisme de Rousseau », Philosophie antique [En ligne], 11 | 2011, mis en ligne le 01 novembre 2018, consulté le 10 décembre 2020. URL : http:// journals.openedition.org/philosant/993; DOI : https://doi.org/10.4000/philosant.993

La revue Philosophie antique est mise à disposition selon les termes de la Licence Creative Commons Attribution - Pas d'Utilisation Commerciale - Pas de Modification 4.0 International. 


\section{LA QUESTION DU PLATONISME DE ROUSSEAU Francesco GREGORIO \\ FNRS-Université de Lausanne}

RESUME. Le platonisme de Rousseau est un thème minoritaire dans l'histoire de la réception de Rousseau. Malgré cela, il s'agit d'une question débattue, encore ouverte et qui a une histoire. On tente ici de faire le point en commençant par une critique de l'historiographie et par une discussion préalable des difficultés que l'interprète rencontre, afin de situer la généalogie des lectures qui font de Rousseau un platonicien. Dans un second temps, on propose un portrait de Rousseau lecteur de Platon, avant de tenter un portrait du platonisme politique de Rousseau.

SUMMARY. Rousseau's Platonism is not a hegemonic issue in the history of the reception of his work. Notwithstanding, this issue is still open to discussion, and has its own history. This paper tries to tackle this point, starting with an appreciation of the historiography and an examination of the difficulties one has to face when settling this issue. The first point is a genealogical sketch of these interpretations which try reading Rousseau as a Platonist. The second point is an analysis of Rousseau himself as a reader of Plato. Finally, we try to specify Rousseau's political Platonism. 



\section{Introduction}

Est-il possible d'identifier et de qualifier un platonisme chez Rousseau ? La première difficulté consiste à distinguer entre le platonisme comme catégorie historiographique générique et les platonismes comme concrétisations situées, objets de l'histoire de la réception de Platon. La catégorie historiographique «platonisme » semble, notamment lorsqu'elle est utilisée pour qualifier les œuvres des philosophes modernes, être pour le moins floue. En tant que catégorie descriptive générique, le platonisme nomme un ensemble d'idées si générales qu'elles finissent par n'avoir aucune pertinence analytique ${ }^{1}$. Ainsi, à un degré élevé de généralité, on peut poser que la distinction de l'âme et du corps, du sensible et de l'intelligible, de l'un et du multiple, le primat de la géométrie, l'unité des savoirs, le lien entre être, valeur et vérité ou encore la dévalorisation épistémologique des sens relèvent du pedigree du platonisme.

Elaborés au fil des traditions intellectuelles, des héritiers de l'Académie de Platon à Plotin, d'Augustin aux néoplatoniciens de la Renaissance, les platonismes appartiennent à l'histoire de la réception de Platon, qui commence avec Aristote. L'hétérogénéité doctrinale et la forme dialogique du corpus platonicien ont servi de base à une multiplicité de platonismes chaque fois centrés sur une sélection de dialogues ${ }^{2}$. Une recherche portant sur la question du platonisme de Rousseau doit ainsi se prémunir contre une application immédiate du platonisme comme catégorie historio-

1. Sur le cas du platonisme de Descartes, voir les résultats ambivalents commentés par Ayers 2008, p. 1-7. Voir aussi Vieillard-Baron 1988, p. 13 : «L'expression de platonisme a pris en philosophie un sens plat, vague et imprécis, surtout lorsqu'elle est employée à propos des philosophes modernes. »

2. Sur les premières interprétations de Platon, voir Tarrant 2000. 
graphique; et ce d'autant plus que les codes de l'historiographie de la philosophie moderne fonctionnent selon une grammaire de la coupure ancien/moderne, reléguant le platonisme du côté de l'ancien ${ }^{3}$. Il faut en outre tenir compte de la pluralité des platonismes transmis par l'histoire de la réception des dialogues platoniciens. Contre une recherche des sources qui se contente d'isoler des ressemblances au niveau doctrinal, voire de réduire un penseur à l'économie de ses plagiats, il faut poser que l'imputation de l'étiquette « platonisme » à un philosophe dépend des précautions prises dans l'élaboration de la comparaison ${ }^{4}$.

Ces précautions sont nombreuses. On peut poser d'une part une comparaison extérieure et factice, fondée sur une ressemblance adventice, et d'autre part une identification du penseur avec le platonisme. Il est en effet possible de penser à Platon (ou à une version du platonisme) en lisant un philosophe, de même qu'il est possible pour un philosophe de se prendre pour Platon - à tout le moins pour son épigone le plus fidèle. Un philosophe peut être influencé par un auteur, sans pour autant laisser de traces visibles de cette influence. De plus, le travail de repérage d'une réception, qui passe avant tout par le recueil des citations, des mentions et usages explicites, ne peut faire l'économie d'une historicisation de l'écriture, en particulier de l'usage de la citation ${ }^{5}$. D'une manière générale, il s'agit là d'une tâche difficile dans le cas de Rousseau : sa pensée est toujours accompagnée, selon une formule de Starobinski, d'un « complément de subjectivité suggérée $\gg^{6}$.

3. Cf. Hutton 2008.

4. Dès 1766, Dom Cajot faisait paraître un ouvrage intitulé Les Plagiats de Monsieur J. - J. Rousseau. Rousseau s'en moquera en parlant du «gros livre de Cachot ». Cf. OC III, p. 1244 et 1249.

5. Il existe au XVIII ${ }^{\mathrm{e}}$ siècle une politique de la citation qui est un enjeu épistémologique majeur. L'auteur est l'équivalent littéraire du cogito cartésien; il se doit de maîtriser son discours sans l'adosser à une économie citationnelle. Ce débat fut particulièrement virulent dans l'histoire de la réception des Essais de Montaigne, lequel Montaigne fut vivement critiqué par Malebranche. Cf. Compagnon 1979, p. 306-313.

6. L'identification du platonisme implique aussi, de la part du lecteur, une interprétation de Rousseau. Dans l'expression «platonisme de Rousseau », aucun terme ne permet $a$ priori de servir d'assise herméneutique. Starobinski reste une référence majeure pour suivre les registres de l'écriture du Genevois : « C'est une œuvre qui, de la réflexion philosophique à l'autobiographie, de la dialectique la plus serrée à l'épanchement lyrique, de la fiction à la législation, joue sur un nombre considérable de registres et occupe une étonnante diversité de dimensions spirituelles. Il est légitime de parler isolément du penseur ou du rêveur, du politique ou du persécuté, du musicien ou du romancier. » Cf. Starobinski 1971, p. 319. 
Peut-on parler d'un platonisme de Rousseau ? Le cas échéant, peut-on dire que Rousseau est platonicien ? Pour y répondre de manière aussi analytique que possible, il faudrait détailler les différentes configurations qui peuvent relever du platonisme dans l'hétérogénéité des livres de Rousseau. Quelles sont les conditions pour attribuer le qualificatif « platonicien » à Rousseau ? Un point est établi : jamais Rousseau ne se déclare platonicien. On ne peut donc se rabattre sur une revendication propre à Rousseau (ce qui ne règlerait rien, tant le labyrinthe autobiographique de Rousseau est déroutant). S'il s'est identifié en imagination aux héros de Plutarque comme exemples de grandes âmes ${ }^{7}$, s'il s'est projeté dans les figures exemplaires de Socrate, de Caton, de Fabricius ou de Jésus, Rousseau n'a jamais déclaré suivre la doctrine de Platon - quelle que puisse être la configuration de cette doctrine. La voie directe d'une filiation revendiquée ou projetée est donc barrée. Ne reste que la voie indirecte, moins assurée. On est contraint d'avancer par triangulation, et ce d'autant plus que d'autres éléments doivent être pris en compte : lire Platon au XVIII ${ }^{\mathrm{e}}$ siècle est une opération qui doit être historicisée et contextualisée. Afin de dégager le terrain, on choisit ici la voie de la critique de l'historiographie, afin de situer cette famille particulière de lectures faites sur l'œuvre de Rousseau. Dans un second temps, je procéderai à un portrait de Rousseau lecteur de Platon. Enfin, je tenterai d'affronter la question du platonisme politique de Rousseau.

\section{Historiographie}

Dans son étude classique, les Méditations métaphysiques de Jean-Jacques Rousseau, Henri Gouhier note: «Penser à Platon en lisant Rousseau peut étonner ${ }^{8} . \gg$ L'étonnement se renforce si l'on se penche sur l'historiographie sur Rousseau. En effet, au long du $\mathrm{XX}^{\mathrm{e}}$ siècle court une tradition historiographique qui s'attache à penser à Platon en lisant Rousseau. On peut situer le point de départ de cette tradition en 1934, avec le livre de Hendel, JeanJacques Rousseau Moralist - l'idée du livre date de 1915 ; un premier projet, avorté, avait pour titre : Rousseau the Platonist. Elle se termine, provisoirement, en 2007, avec le livre de Williams, Rousseau's Platonic Enlightenment. Notons que cette tradition reste minoritaire dans le champ des études rousseauistes, comme en témoigne la quasi-absence d'études sur ce sujet dans les Annales de la Société Jean-Jacques Rousseau.

7. OC I, p. 1134.

8. Gouhier 1970, p. 133. 
Il existe ainsi dans l'historiographie sur Rousseau une famille d'interprétations qui cherche à soutenir un certain nombre de thèses herméneutiques que l'on peut en première approche résumer de la manière suivante : Rousseau est un platonicien, à savoir : il existe sous la surface, de manière implicite, une forte présence de Platon chez Rousseau ; Rousseau professe un platonisme, à savoir : il est possible d'isoler des philosophèmes de Platon dans les termes de la philosophie de Rousseau ; Rousseau platonise, à savoir : il existe un registre dans l'écriture de Rousseau qui renvoie à un fonds platonisant. Ces interprétations laissent leurs catégories d'analyse imprécisées, se limitant à parler de platonisme implicite, que la sagacité de l'interprète doit mettre au jour. De plus, ces lectures s'appuient souvent sur une version du platonisme pour construire leur comparatisme. Or la catégorie « platonisme » est loin d'être stabilisée'. Aujourd'hui, tout comme dans la longue histoire de la réception de Platon, il existe, au-delà d'une certaine stabilisation paradigmatique offerte par les recherches historico-philologiques, une pluralité de platonismes. En d'autres termes, la question de l'imputation d'un platonisme à Rousseau ne peut faire l'économie d'une détermination critique des catégories d'analyse mobilisées dans la recherche, l'identification et la spécification d'un platonisme chez Rousseau. Dans le cas du platonisme politique, la chose est d'autant plus délicate que les catégories relevant du politique («politique », « libéralisme», « autoritarisme » ou « collectivisme », par exemple) fonctionnent selon une sémantique qu'il faut toujours situer pragmatiquement et historiciser ${ }^{10}$.

Commençons par le rôle que le platonisme de Rousseau a joué dans l'histoire de la critique. Dans l'histoire des lectures de Rousseau au XX siècle, le thème historiographique du platonisme de Rousseau doit être reconduit au grand conflit des interprétations qui s'est développé sur l'œuvre de Rousseau. Cet ensemble de discours a proliféré dès le vivant même de Rousseau ${ }^{11}$. En effet, l'histoire de l'interprétation de l'œuvre de Rousseau commence avec Rousseau lui-même. Ce dernier a configuré un premier pli herméneutique et indiqué rétrospectivement comment il voulait être lu. Le fil rouge de ces autocommentaires est un avertissement que certains pour-

9. Cf. Wallach 2001, p. 17-40.

10. En 1929 déjà, Wright pointait cette difficulté : « The Contrat Social is meant for neither individualist nor absolutist. » (Wright 1929, p. 103). Depuis les travaux des historiens de l'École de Cambridge, en premier lieu Pocock et Skinner, on sait que le langage politique doit être situé dans son contexte d'énonciation. $C f$. Hampsher-Monk, Tilmans \& Van Vree 1998 ; Brett, Tully \& Hamilton-Bleakley 2006.

11. Cf. Trousson 1977. 
raient déjà imputer à une structure «platonicienne ». En effet, Rousseau insiste sur l'unité de principe de sa philosophie : son lecteur doit bien voir, en deçà des différences de surface, dues aux contingences d'une pensée au travail. Par exemple, l'écho de Diderot dans le naturel de Rousseau donne une inflexion stylistique à la parole de ce dernier : « car pour Diderot je ne sais comment toutes mes conférences avec lui tendoient toujours à me rendre satyrique et mordant plus que mon naturel ne me portoit à l'être ${ }^{12}$. » Toujours dans les Confessions, Rousseau lit ses propres textes sous le même angle d'une cohérence au-delà des apparences hétérogènes : «Tout ce qu'il y a de hardi dans le Contrat social étoit auparavant dans le Discours sur l'inégalité; tout ce qu'il y a de hardi dans l'Émile étoit auparavant dans la Julie $^{13}$. » De même, dans sa Lettre à C. de Beaumont, archevêque de Paris, Rousseau souligne: «J'ai écrit sur divers sujets, mais toujours dans les mêmes principes : toujours la même morale, la même croyance, les mêmes maximes, et, si l'on veut, les mêmes opinions ${ }^{14}$. » Mais il semble que les yeux peu «platoniciens » de ses contemporains n'aient pas su faire le tri entre la surface et la profondeur du principe qui anime la pensée de Rousseau. Rousseau se plaint que l'on porte des jugements opposés sur ses livres, qu'on fasse de l'auteur un homme à paradoxes : « Ainsi va flottant le sot public sur mon compte ${ }^{15}$.

La réception de l'œuvre de Rousseau ne va pas suivre le mode d'emploi que l'auteur même propose ${ }^{16}$. Au lieu de chercher les « mêmes principes » sur lesquels les textes de Rousseau sont construits, l'œuvre sera prise dans la querelle de la modernité politique, radicalisée par le jacobinisme révolutionnaire durant la révolution française. L'historiographie rousseauiste a ainsi dégagé plusieurs visages de Rousseau : révolutionnaire, individualiste, libéral, antilibéral, totalitaire, conservateur, républicain, romantique, collectiviste $^{17}$. Lanson est peut-être le premier interprète à prendre au sérieux les affirmations de Rousseau concernant l'unité de principe de sa pensée ${ }^{18}$. Se

12. $O C$ I, p. 405 , note.

13. OC I, p. 407.

14. $O C$ IV, p. 928. Rousseau y est revenu souvent. Voir par exemple $O C \mathrm{I}$, p. 930 ; $O C \mathrm{I}$, p. 31 .

15. Ibid. Dans l'Émile, Rousseau avertit son lecteur «effrayé » : « Lecteurs vulgaires, pardonnez-moi mes paradoxes : il faut en faire quand on réfléchit. » Cf. OC IV, p. 321 et 323.

16. Cf. Trousson 1977.

17. Pour une première approche de l'histoire de la réception, voir Ottmann 2006, p. 503-507.

18. Cf. Gay 1964. 
dégage une consigne herméneutique simple : il faut lire tout Rousseau, tenir ensemble toute l'œuvre, et éviter les sélections partielles et instrumentales destinées à un discours plus idéologique que scientifique. Prendre Rousseau au sérieux signifie le lire comme un philosophe.

Mais l'image du Rousseau philosophe est elle aussi démultipliée. On cherche en Rousseau un disciple ou un précurseur : philosophe de l'existence, penseur du moi et du sentiment, phénoménologue, d'une part ; philosophe politique de la vertu républicaine, de la révolution et de la science, et de l'ethnologie d'autre part. Avec Cassirer puis Starobinski, les études sur Rousseau atteignent leur maturité. Cassirer propose en 1932 une lecture de Rousseau à partir de la lecture qu'en fit Kant. Il tente lui aussi de trouver le centre unifiant de la pensée de Rousseau, à partir de la thématisation du « problème » Rousseau ${ }^{19}$. Le livre de Starobinski, La Transparence et l'obstacle, tente lui aussi une lecture unificatrice à partir de la structure entre médiat et immédiat qui traverse toute l'œuvre de Rousseau, selon l'axiome suivant : Rousseau a besoin du médiat pour restaurer l'immédiat. En particulier, au niveau de l'écriture, Rousseau a besoin de la médiation du langage pour dire qu'il ne veut pas du langage. Le livre de Starobinski n'aborde pas la question du platonisme de Rousseau. Tout au plus accorde-t-il à Rousseau une pratique accessoire du platonisme, relevant de ce que Starobinski nomme un «platonisme traditionnel $\gg^{20}$ : « Rousseau n'a pas toujours proclamé la "vérité des sensations". Dans les moments où il "platonise", il discrédite les sens comme des puissances d'erreur ${ }^{21}$. 》

Le livre de Victor Goldschmidt, Anthropologie et politique, se présente comme un grand commentaire du Premier et surtout du Second Discours, que Goldschmidt lit comme le texte le plus philosophique de Rousseau. La quarantaine de références à Platon qu'on y trouve sont très secondaires. Ce sont dans la majorité des rapprochements opérés pour clarifier une position, inscrire une thèse de Rousseau dans la tradition de pensée, même si

19. Cassirer 1932. Cassirer rapproche Rousseau de Platon (et en particulier de la République) en l'éloignant de l'anthropologie politique d'Aristote. Mais c'est finalement Kant qui offre à Cassirer la clé d'une lecture philosophique de Rousseau. Le platonisme de Rousseau est donc médiatisé par le platonisme épistémologique et éthique de matrice kantienne (l'idéal n'est plus ontologiquement indépendant, opposé à l'expérience, mais en est une composante fondamentale).

20. Starobinski 1971, p. 97.

21. Starobinski 1971, p. 41, note 1, avec un renvoi à $O C$ IV, p. 1092. 
l'on rencontre les expressions « platonisme méthodologique » et « platonisme implicite $\gg^{22}$.

Plus intéressante pour notre question est la réflexion conclusive de Goldschmidt sur le dualisme du système de Rousseau ${ }^{23}$. Il entend par là la manière qu'a Rousseau de réfléchir à partir de thèses opposées, comme par exemple: «les hommes sont méchants» et «cependant l'homme est naturellement bon ». On peut aussi penser à la première phrase du Contrat social. Selon Goldschmidt, «c'est en cela que consiste, fondamentalement, le "platonisme de Rousseau" ${ }^{24}$. Si ce trait détermine en effet l'écriture de Rousseau, on ne voit pas en quoi ce dualisme est pertinent pour qualifier la réception de Platon chez Rousseau. Il est d'ailleurs plus probable que ce trait du style de Rousseau soit à rapprocher du style baroque, friand de structures oppositionnelles. Goldschmidt en reste au plan des principes du système de Rousseau. Il n'a pas cherché à spécifier les lectures et les usages que Rousseau a faits de Platon.

Cette manière de penser par couples d'opposés est aussi soulignée par Burgelin ${ }^{25}$. En soulignant la lecture singulière que Rousseau fait des Oratoriens platonisants (avant tout Malebranche), Burgelin réduplique cette structure oppositionnelle en proposant de qualifier de manière paradoxale le platonisme de Rousseau en forgeant l'expression «platonisme du cœur $\gg^{26}$, qu'il applique surtout à la petite communauté de la Nouvelle Héloïse.

Du côté des grands interprètes de la pensée politique de Rousseau, la situation est là aussi ambivalente. Dans son livre classique sur Rousseau, Robert Derathé signale d'une part : « Quoiqu'il ne soit pas facile de déterminer quelles ont été les lectures politiques de Rousseau, on peut admettre en effet qu'en dehors de Platon, l'un de ses auteurs favoris, il a lu les penseurs qui faisaient autorité à son époque. » Mais ce constat est aussitôt suivi d'une exclusion : «On ne trouvera donc point dans ce travail une étude complète des sources de la pensée politique de Rousseau, puisque nous avons dû laisser de côté l'influence pourtant décisive de Platon et celle non

22. Cf. Goldschmidt 1974, p. 35 et 629.

23. Ibid. p. 778 .

24. Ibid.

25. Cf. Burgelin 1973, p. 2.

26. Cf. Burgelin 1973, p. 173 et 405. 
moins importante de Montesquieu ${ }^{27}$. $\gg$ La possibilité d'une réception du platonisme politique chez Rousseau est donc exclue de l'analyse ${ }^{28}$.

En revanche, le platonisme apparaît comme un opérateur d'unification qui va de Vaughan en 1915 à Williams en 2007. Là aussi, il convient de distinguer les différents types de platonisme attribués à Rousseau, en suivant la piste de ce que l'on pourrait appeler une politique de la qualification du platonisme. En effet, le plus souvent, le platonisme attribué à Rousseau peut être spécifié par une détermination complémentaire. Il y a d'abord ce que l'on pourrait nommer un platonisme stratégique, dans la mesure où il sert à unifier et à orienter l'ensemble de la philosophie de Rousseau. Dans l'histoire de la recherche portant sur l'œuvre et la pensée de Rousseau, la question du platonisme est configurée pour la première fois par Vaughan, qui livre en 1915 une édition des textes politiques de Rousseau. On peut parler ici de la naissance d'un platonisme stratégique, dans la mesure où il permet à Vaughan de configurer une lecture unitariste de la pensée de Rousseau, en suivant le thème de l'éducation publique. Vaughan lit les textes politiques de Rousseau en cherchant à neutraliser les lectures partiales qui se déchirent entre un Rousseau libéral et un Rousseau antilibéral. Il s'agit moins de les réconcilier que d'instruire le conflit entre ces lectures en suivant les textes à partir de la question de l'éducation publique ${ }^{29}$. Dans son introduction et ses commentaires aux écrits politiques de Rousseau, Vaughan propose un cadre herméneutique qui s'articule autour de l'article Économie politique, rédigé pour l'Encyclopédie en 1755 et dont on sait qu'il est fortement influencé par la lecture que Rousseau fit des Lois de Platon. Selon Vaughan, à partir de ce texte, Rousseau n'est plus disciple de Locke, mais de Platon ${ }^{30}$. Avec Vaughan, le platonisme apparaît comme un prétendant possible comme principe de lecture de la pensée de Rousseau. Mais en introduisant le platonisme comme une rupture dans le développement de

27. Derathé 1970, p. 25, 27. On trouve un même geste de valorisation et d'exclusion dans le cas de Platon cette fois, dans un article que Derathé a consacré à l'influence de Montesquieu sur Rousseau. Montesquieu est «l'homme auquel il (scil. Rousseau) doit plus qu'à tout autre penseur, à l'exception toutefois de Platon » (Derathé 1955, p. 373.) On ne peut s'empêcher de voir chez Derathé une stratégie d'évitement de la question du platonisme de Rousseau.

28. À un premier niveau général, on peut poser que le platonisme politique décrit un ensemble de thèses que l'on trouve dans les dialogues platoniciens suivants : Politique, République, Lois, auxquels il faudrait ajouter Gorgias, Protagoras, Criton et la Lettre VII.

29. Vaughan 1915, I, p. 5.

30. Vaughan 1915, I, p. 236. Pour un relevé du platonisme (chrétien) chez Locke, voir Nuovo 2008. 
l'œuvre de Rousseau, Vaughan coupe Rousseau en deux moitiés : Rousseau fut d'abord individualiste, «moderne ». Il fut ensuite collectiviste, « ancien ». Le platonisme de Rousseau rejoue ainsi, à son niveau, la querelle des Anciens et des Modernes.

En 1915, Hendel a en tête le projet d'un livre, Rousseau the Platonist. Il y renoncera ${ }^{31}$. Sur ce projet avorté, Hendel construira peu à peu sa grande monographie sur Rousseau qui paraîtra en 1934 sous le titre Jean-Jacques Rousseau Moralist. Pour Hendel, le platonisme est une composante importante de l'œuvre de Rousseau. Certes Rousseau n'est pas un scholar. Mais la lecture de Platon est un élément important dans la critique que Rousseau fait de la société et dans le projet d'une politique républicaine. Chez Hendel, le platonisme de Rousseau alimente les éléments républicains de sa philosophie. Pour le montrer, Hendel suit la genèse de la pensée de Rousseau, montrant ainsi que le platonisme de Rousseau fait partie intégrante de l'ensemble de l'œuvre. On a alors affaire cette fois à un platonisme stratégique complet : Platon ne coupe pas Rousseau en deux, il l'accompagne tout au long de sa vie de penseur.

On peut considérer la monographie de Williams, Rousseau's Platonic Enlightenment comme un aboutissement de cette famille de lectures qui cherchent à isoler un platonisme stratégique chez Rousseau. Williams poursuit la stratégie herméneutique qui voit dans l'identification d'un platonisme chez Rousseau la clé d'unification de sa philosophie. Rousseau est vu comme un très grand platonicien, le plus cohérent de l'ère moderne ${ }^{32}$. Williams fait une lecture platonicienne de Rousseau en proposant une scénographie à la fois dramatisante et quelque peu simplificatrice : Rousseau trouve un allié en Platon contre Hobbes et les Philosophes, contre le matérialisme et le sensualisme du XVIII ${ }^{\mathrm{e}}$ siècle $^{33}$. Opérant avec des catégories d'analyse qui relèvent du pedigree du platonisme, Williams dramatise l'opposition entre sensible et intelligible : d'un côté l'empirisme, de l'autre l'idéalisme. Avec de telles catégories historiographiques, Williams peut mettre en scène une nouvelle histoire sainte du platonisme qui met dans le même groupe Descartes, Cudworth, Leibniz et Malebranche, contre le ma-

31. Hendel 1934, p. vii : «An extensive work, 'Rousseau the Platonist', was then projected; but, on the wise counsel of my teacher and friend Professor Norman Kemp Smith, this was given up for a time (...) ». On aimerait en savoir plus sur les raisons qui ont motivé le « sage conseil » de Kemp Smith. Voir la recension très critique de Schinz 1934.

32. Voir sa formule emphatique : «Indeed, my thesis is that he is among the greatest and most consistent Platonists of the modern era. » (Williams 2007, p. 94.)

33. Cf. Williams 2007, p. 1-59. 
térialisme de La Mettrie, d'Holbach, d'Helvétius et de Diderot ${ }^{34}$. Pour Williams, le platonisme se concentre dans une doctrine qui soutient la foi en Dieu, le libre arbitre, l'immortalité de l'âme et les idées transcendantes. Du côté du comparant, Williams s'appuie sur le platonisme de matrice kantienne formalisé par T.K. Seung ${ }^{35}$. La monographie de Williams est intéressante dans la mesure où elle tente de généraliser le modèle herméneutique de Hendel. Mais là est aussi le problème d'une telle approche : cette mise au jour du platonisme s'appuie d'une part sur une straw man fallacy qui simplifie les positions des adversaires. Elle n'historicise pas les régimes de réception (qui sont aussi des régimes de perception) propres à Rousseau.

À côté de cet usage stratégique du platonisme chez Rousseau, on trouve une autre famille de lectures qui mobilise un platonisme sectoriel, ou minoritaire. C'est par exemple le cas de Gouhier, qui parle de « platonisme périphérique »: «on ne dira donc pas que le platonisme de Rousseau est superficiel mais périphérique, parce qu'une influence peut être assez profonde sans être pourtant reconnaissable au principe et, par suite, au centre d'une pensée en train d'inventer sa propre philosophie ${ }^{36}$. » Du point de vue des catégories d'analyse, Gouhier propose une image plus complexe : le schème aristotélicien du centre et de la périphérie vient modaliser et minoriser en partie le schème platonicien de la surface et de la profondeur. Gouhier avance deux arguments principaux. D'une part, la lecture de Platon a joué un rôle de déclencheur pour Rousseau. Il lui a permis de prendre de la distance par rapport à la culture de son temps, et surtout contre ses « amis » philosophes, Helvétius, d'Holbach, Diderot. D'autre part, certains thèmes et images platoniciens innervent le Rousseau penseur de l'amour et de la solitude. Malgré les concessions faites à la prégnance de Platon chez Rousseau, Gouhier maintient que la structure fondamentale de la philosophie de Rousseau est fondamentalement moderne, lockéenne ${ }^{37}$.

Résumons. Il y a une politique du platonisme dans l'histoire de la recherche sur Rousseau. Une première famille de lectures porte sur la mobilisation du platonisme comme le principe d'unité de la philosophie de Rous-

34. On a pu identifier aussi un matérialisme tempéré chez Rousseau. Cf. Vernes 2009.

35. Cf. Seung 1996 et Williams 2007, p. 95. Le Platon de Williams est donc celui, kantien, de Seung, avec lequel il construit le platonisme de Rousseau.

36. Cf. Gouhier 1970, p. 149.

37. Cf. Gouhier 1970, p. 133. 
seau. Une seconde famille de lectures isole un platonisme important mais secondaire, qualifié tout à tour d'implicite ou périphérique ${ }^{38}$.

Ce petit passage en revue de la situation historiographique concernant la question du platonisme de Rousseau nous amène à une première conclusion peu spectaculaire mais utile : il y a une pluralité de platonismes de Rousseau dans l'historiographie. Il existe un platonisme utilisé comme catégorie analytique positive, qui se dédouble en un platonisme stratégique d'ensemble et un platonisme secondaire ou sectoriel. Par ailleurs, et de façon dominante dans le cas de l'histoire de la pensée politique, il existe un platonisme valorisé mais exclu de l'analyse, et un platonisme utilisé comme catégorie analytique négative, qui s'inscrit dans le conflit entre politique des Anciens et politique des Modernes.

\section{Rousseau lecteur de Platon}

Est-il possible de se frayer une voie dans cette forêt des platonismes historiographiques? Une leçon que l'on peut tirer du pluralisme des positions historiographiques est que le simple repérage doctrinal ne suffit pas. La proposition « Rousseau est un philosophe qui lit Platon» demande à être spécifiée. On ne peut se contenter d'une Begriffsgeschichte. Une possibilité serait de prendre en compte l'histoire intellectuelle, l'histoire de l'hellénisme et plus généralement l'anthropologie des mondes savants au $\mathrm{XVIII}^{\mathrm{e}}$ siècle $^{39}$. Le travail intellectuel de Rousseau doit être inscrit dans la situation de la philosophie en France à son époque. La philosophie comme discipline, le philosophe comme auteur émergent comme des enjeux culturels qui instruisent un conflit de légitimation en dehors de toute stabilisation institutionnelle dans l'Université. Le philosophe se transforme en auteur qui revendique une valeur littéraire ${ }^{40}$.

Ce lien entre philosophie et littérature est évident dans le cas de Rousseau. Non seulement dans le cas de Rousseau écrivain, dont Starobinski a fourni des analyses magistrales, mais aussi dans le cas de Rousseau lecteur. C'est par exemple le cas de sa lecture de Montesquieu, puisque Rousseau ne dissocie pas le penseur de l'écrivain. Montesquieu est un grand penseur

38. On trouve dans la littérature d'autres variantes: platonisme caché et modifié (Cooper 2002), platonisme oscillant (De Luise 2000, p. 490).

39. Le cadre général de cette approche est fourni par l'entreprise collective dirigée par Jacob 2007-2011. Sur le XVIII ${ }^{\mathrm{e}}$ siècle, $c f$. Ribard 2003.

40. Cf. Ribard 2003, p. 7-29. 
précisément parce qu'il est un grand écrivain ${ }^{41}$. Rousseau est très sensible à la qualité littéraire de l'expression philosophique. On peut poser l'hypothèse que ce lien entre la pensée et son expression a joué un rôle dans les lectures de Platon et dans les quelques fragments de Platon que Rousseau a traduits. La lecture et la traduction des grands auteurs s'inscrivent dans une pratique stylistique, dans laquelle il n'est pas possible de séparer le philosophique du littéraire ${ }^{42}$.

Demandons-nous d'abord comment Rousseau lit Platon. Le premier point à souligner est que Rousseau est un autodidacte, formé hors de l'espace académique. L'autodidactisme est un accès solitaire et indépendant qui structure l'accès de Rousseau à la pensée ${ }^{43}$. Il ne s'inscrit pas dans un Platonbild particulier. Au contraire, il fera au fil de ses lectures un montage hybride de plusieurs types de platonismes que nous essayerons de spécifier.

L'antiquité et le christianisme constituent deux références culturelles essentielles pour la philosophie de Rousseau ${ }^{44}$. Parmi les auteurs antiques, Platon est très important. Un simple relevé statistique le prouve : cité cinquante-huit fois ${ }^{45}$, Platon vient en troisième position, après la Bible (quatre-vingt-six) et Plutarque (soixante-dix); suivent Buffon (cinquante occurrences), Montaigne (trente-trois), Aristote (vingt-six) et Montesquieu (vingt-cinq). Des cinquante-huit occurrences relevées par Richebourg, une douzaine se rapportent à la musique. De manière générale, l'étude des citations est décevante et ne permet pas de fonder une interprétation du platonisme de Rousseau. Il faut alors poser un problème bien plus difficile à trancher qu'un recensement d'occurrences: le régime de lecture que Rousseau pratique sur Platon est spécifique. Il entrâne une redéfinition des codes de lecture culturellement admis, aussi bien du christianisme que de l'antiquitét ${ }^{46}$. En ce sens le Platon de Rousseau est le produit singulier du régime de lecture de Rousseau.

41. Cf. Ehrard 1997, p. 62-63.

42. Rousseau lit et traduit pour mieux écrire et pour mieux penser. Dans l'Émile, il commente son propre conflit entre ses idées (cohérentes) et ses expressions (inévitablement contradictoires en raison de la pauvreté de la langue). Cf. $O C$ IV, p. 345, note.

43. Cf. Ribard 2003, p. 115-121.

44. Pour une vision panoramique, $c f$. Touchefeu 1999, p. 35-36, qui montre notamment comment le montage entre culture antique et culture théologique était bien établi dans la Genève du jeune Rousseau, en raison de l'essor de l'édition genevoise et de sa diffusion dans la rhétorique politique de son temps.

45. Cf. Richebourg 1934 .

46. Dans le cas du christianisme, la théologie (politique) et la christologie de Rousseau sont en effet très idiosyncrasiques : le refus de l'universalisme chrétien et le refus de la croix 
On sait que Rousseau est un grand lecteur, en dépit des nombreuses attaques que l'on trouve dans ses textes contre les livres et la lecture ${ }^{47}$. Mais on trouve chez Rousseau une diversité de régimes de lecture. Il y a d'abord la lecture-compensation, qui cherche à se réfugier dans le livre. Rousseau parle alors d'une fureur de lire, accompagnée d'une singulière capacité de projection dans l'imaginaire littéraire ${ }^{48}$. Lectures compulsives, désordonnées. Lectures de dilettante: mathématiques, histoire, musique, sciences, littérature, échecs ${ }^{49}$. À Turin, en 1728, Rousseau se lie avec l'abbé Gaime. Selon Masson, c'est Gaime qui apprit à Rousseau à mettre de l'ordre dans ses lectures et à se faire des extraits ${ }^{50}$. Quoi qu'il en soit, il est certain que Rousseau a pris cette habitude de se faire des cahiers d'extraits. Certains ont été retrouvés dans les Manuscrits de Neuchâtel. Malheureusement, le plus ancien cahier d'extraits dont nous disposons date de 1750 . Nous n'avons donc pas accès aux cahiers des années de formation. Mais on peut supposer que Rousseau s'est fait de nombreux extraits des ouvres de Platon, en particulier de la République et des Lois ${ }^{51}$. Sur le premier feuillet des CEuvres de Platon, dans la traduction de Ficin, Rousseau a ainsi noté quelques traductions, issues de la République, des Lois et du Phédon ${ }^{52}$. Dans sa notice, Bernard Gagnebin rapporte une phrase que Rousseau a écrite sur la page de garde de son édition : «On a beau renier l'idole qu'on sert, elle ne meurt point $^{53}$. $\gg$ Il existe aussi un parergon à la Lettre à d'Alembert, un petit texte d'une quinzaine de pages intitulé De l'imitation théatrale. Dans l'avertissement à ce texte, Rousseau note: «Ce petit écrit n'est qu'une espèce d'extrait de divers endroits où Platon traite de l'Imitation théâtrale. Je n'y ai gueres d'autre part que de les avoir rassemblés et liés dans la forme d'un discours suivi, au lieu de celle du Dialogue qu'ils ont dans l'original ${ }^{54}$. »

donnent une idée de la manière dont Rousseau retravaille les massifs culturels. $C f$. Starobinski 1971, p. 87-90.

47. $O C$ IV, p. XXII-XXVI.

48. $O C$ I, p. 39-40.

49. Richebourg 1934, p. 27-30, 40.

50. Richebourg 1934, p. 17.

51. Dans une note à son édition de la première version de l'Émile (ms. Favre), Spink écrit : « Rousseau avait copié de nombreux passages de la République. » (OC IV, p. 1299, n. 2 de la p. 250.)

52. Cette dizaine de phrases se trouve dans l'Appendice VI de l'édition de la Pléiade (OC V, p. 1297-1298).

53. $O C \mathrm{~V}$, p. CCCVI.

54. $O C \mathrm{~V}, \mathrm{p} .1195-1211$ 
Rousseau commente lui-même ces différents régimes de lecture, en jouant sur le registre d'une comparaison entre différents régimes de lecture. Il écrit qu'il ne lit plus comme un Français, mais comme un Genevois : « Je reviens à ce goût de la lecture qui porte les Génevois à penser. (...) Le François lit beaucoup ; mais il ne lit que les livres nouveaux, ou plutôt il les parcourt, moins pour les lire que pour dire qu'il les a lus. Le Génevois ne lit que les bons livres; il les lit, il les digere : il ne les juge pas, il les sait ${ }^{55}$. » Aux Charmettes, Rousseau organise ses lectures :

En lisant chaque Auteur, je me fis une loi d'adopter et suivre toutes ses idées sans y mêler les miennes ni celles d'un autre, et sans jamais disputer avec lui. Je me dis, commençons par me faire un magasin d'idées, vrayes ou fausses, mais nettes, en attendant que ma tête en soit assez fournie pour pouvoir les comparer et choisir. (...) Pour avoir commencé tard à mettre en exercice ma faculté judiciaire, je n'ai pas trouvé qu'elle eut perdu sa vigueur, et quand j'ai publié mes propres idées, on ne m'a pas accusé d'être un disciple servile, et de jurer in verba magistri ${ }^{36}$.

Rousseau a dressé une liste de ses lectures dans le Verger de Mme de Warens, un poème de 156 vers $^{57}$. Il n'est pas original dans le choix de ses lectures : «Les vrais maîtres de Rousseau ne doivent pas être cherchés dans les sentiers écartés, mais dans les chemins battus. Ce sont Hobbes et Grotius, ce sont aussi Pufendorf et Barbeyrac; mais ce sont surtout Locke, Montesquieu et Platon ${ }^{58}$. $\gg$ On peut y ajouter le Télémaque de Fénelon dont on sait la place centrale qu'il occupera dans la formation d'Émiles9. C'est aussi le livre le plus lu au XVIII ${ }^{\mathrm{e}}$ siècle, après la Bible.

Rousseau ne sait pas le grec. Tout au plus en est-il frotté, comme on peut l'apprendre dans sa correspondance. En 1749, Rousseau est à Paris. Son conflit avec Rameau au sujet des Muses galantes est tout récent. Affecté, haineux, Rousseau se réfugie alors dans le travail de rédaction des articles sur la musique qu'il a promis à Diderot pour l'Encyclopédie. Le 27 janvier 1749, il écrit à madame de Warens: «Je bouquine, j’apprens le Grec. Chacun a ses Armes; au lieu de faire des chansons à mes Ennemis, je

55. $O C$ II, p. 660.

56. $O C$ I, p. 237-238. Il faut relier cette déclaration avec la politique de la citation au XVIII ${ }^{\mathrm{c}}$ siècle. Voir supra note 5.

57. OC II, p. 1123-1129.

58. Vaughan 1915, II, p. 8, cité et traduit par Derathé 1970, p. 25.

59. OC IV, p. 762. 
leur fais des articles de Dictionnaire ${ }^{60}$. $\gg$ Mais en 1757 il écrit dans une lettre à Tronchin : «Je ne sais point de grec. » Il lira de nombreux auteurs grecs en traduction française ${ }^{61}$.

Rousseau sera meilleur latiniste ${ }^{62}$. Ses compétences en latin lui permettent de lire le Platon de Ficin, d'en traduire des extraits. Il traduira en 1754 le livre I des Histoires de Tacite ${ }^{63}$. Nous savons que la traduction latine des œuvres de Platon qu'il a utilisée fut celle de Ficin, corrigée par Simon Grynaeus ${ }^{64}$. L'étude des exemplaires de Rousseau de la traduction de Ficin, aujourd'hui au British Museum, a été faite par Silverthorne ${ }^{65}$. Si l'on suit la piste des annotations marginales de Rousseau faites sur sa copie, cinq dialogues semblent l'avoir intéressé : Apologie de Socrate, Phédon, Politique, République et Lois. Grossièrement, trois thèmes se trouvent ainsi focalisés : la figure de Socrate, l'immortalité de l'âme et la question politique.

La posture du sage Socrate est un thème qui déborde largement l'œuvre de Rousseau et qui réunit, sous des éclairages différents, Rousseau et ses anciens amis de l'Encyclopédie. Au-delà, l'image de Socrate appartient aussi à la vie culturelle du XVIII ${ }^{\mathrm{c}}$ siècle $^{66}$. Mais le Socrate de Rousseau n'est pas celui des Encyclopédistes.

Cette même année durant laquelle Rousseau s'essaie au grec ancien, en 1749, le 24 juillet Diderot est arrêté, emprisonné dans la tour du château de Vincennes. Un philosophe enfermé par un pouvoir inique : il n'en faut pas plus pour mobiliser l'image de Socrate. Diderot lui-même, derrière les barreaux, traduit l'Apologie de Socrate ${ }^{67}$. Rousseau rend souvent visite à son ami. Il est possible qu'avec son aide il ait testé son grec sur le texte de

60. Leigh $1965, \mathrm{n}^{\circ} 146,27$ janvier 1749.

61. Cf. Richebourg 1934, p. 201-202.

62. Cf. $O C$ I, p. 97 ; voir aussi p. 239 : « À force de tems et d'exercice je suis parvenu à lire assez couramment les auteurs latins, mais jamais à pouvoir ni parler ni écrire dans cette langue. 》

63. Cf. von Stackelberg 1958. La traduction de Rousseau a été éditée dans VolpilhacAuger 1995.

64. Divini Platonis operum a M. Ficino tralatorum omnia emendatione et ad Graecum codicem collatione S. Grynaei summa diligentia repurgata, 5 vol., Lugduni, J. Tornaesius, 1550,5 vol.

65. Cf. Silverthorne 1973.

66. Sur les différentes figures de Socrate au XVIII siècle, $c f$. Raschini 2000, p. 211-321.

67. Diderot, Correspondance, IV, 161 (lettre à Sophie Volland du 23 septembre 1762). On peut lire la traduction de Diderot dans Trousson 1969, p. 141-184. 
Platon $^{68}$. L'accès au texte est motivé par une situation politique, par une urgence de résistance projetée sur la figure du sage philosophe. Cette expérience trouve un écho dans le premier Discours. La longue citation de l'Apologie donnée par Rousseau est tirée de la traduction de Diderot - à laquelle il a peut-être collaboré69.

Diderot stylise son arrestation sous la guise de Socrate, un « Socrate » qui lui-même traduit le Socrate de Platon, nouant la vie politique et la vie intellectuelle dans un geste de résistance. Si Rousseau y fut sensible, son Socrate n'est pas celui de Diderot. Le point de divergence porte sur la question de la stylisation de la figure de Socrate : pour Rousseau, Diderot mobilise Socrate au sein d'un acte de résistance culturelle, au service de la culture et du savoir. Rousseau en revanche veut incarner Socrate comme forme de vie opposée à la culture de son temps, mettant au centre l'ignorance comme levier critique de tous les savoirs. La différence passe entre une critique modérée et une critique radicale de la société. Rousseau incarne l'atopie culturelle de Socrate, et ses adversaires sont tous des sophistes: « Lisez, Monsieur, les Dialogues de Platon et vous trouverez que vous faisiez le rôle $\mathrm{du}$ sophiste orgueilleux et vôtre adversaire celui de Socrate. Il vous écrasoit modestement d'argumens terribles auxquels vous répondiez avec beaucoup de hauteur des injures pour des raisons ${ }^{70}$. $\gg$ Rousseau semble non pas tant lire Platon que se projeter dans la structure dramaturgique des Dialogues platoniciens - on retrouve le complément de subjectivité suggérée isolé par Starobinski, qui incarne une posture platonicienne contre les antiplatonismes des Philosophes ${ }^{71}$. De même, on retrouve ce lien, typique de la situation de la philosophie et des philosophes en France au XVIII ${ }^{\mathrm{e}}$ siècle, entre travail de la pensée et travail de la forme littéraire de la pensée.

Rousseau lit donc Platon, s'en fait des extraits, mobilise et radicalise la figure critique de Socrate en martyr de la vérité. Mais il rencontre aussi Platon de manière indirecte, chez les auteurs modernes qu'il lit. Quelques

68. Conjecture de Touchefeu 1999, p. 99. Rousseau aurait pu utiliser deux traductions françaises existantes, celle de P. Giry (1643) ou celle de Dacier (1699). Une autre solution eût été de s'appuyer sur la traduction latine de Ficin, que Rousseau utilisait par ailleurs.

69. Seznec 1957, p. 119-120 et Trousson 1967, p. 54 sqq.

70. OC II, p. 1329. Pour Rousseau, les Philosophes parisiens sont tous des sophistes, $c f$. Williams 2007, p. 89-92.

71. Dans ce jeu de miroirs socratiques, il est remarquable que pour Diderot (dans son article «Philosophie socratique » rédigé pour l'Encyclopédie), Rousseau n'est pas du tout Socrate, mais son contraire, Timon le misanthrope : «Cet homme crut qu'il fuyait la société de ses semblables, parce qu'ils étaient méchants. Il se trompait, c'est que lui-même n'était pas bon. » (Cité par Ribard 2003, p. 81.) 
noms doivent ici être rappelés. Le premier est celui de Marsile Ficin, auteur de la traduction de Platon en latin. De manière étonnante cependant, le nom de Ficin est totalement absent chez Rousseau. Il est donc difficile de documenter des éléments d'un platonisme ficinien présents chez Rousseau. Néanmoins, il est possible de supposer que la lecture ficinienne du Banquet eut pour lui de l'importance. On trouve en effet dans la Nouvelle Héloïse une mobilisation de l'amour platonique. Rousseau indique dans une note de son roman : «La véritable philosophie des Amans est celle de Platon; durant le charme ils n'en ont jamais d'autre. Un homme émû ne peut quitter ce philosophe; un lecteur froid ne peut le souffrir ${ }^{72}$. » La reconstruction la plus convaincante en a été proposée par De Luise et Farinetti qui proposent comme point focal la question du bonheur ${ }^{73}$. Selon les auteurs, on trouve dans la Nouvelle Héloïse trois parcours de recherche du bonheur, qui se présentent comme trois modèles théoriques figurés dans la prose des lettres des amants. Il s'agit des bonheurs solitaire, politique et affectif. C'est dans le bonheur affectif axé sur l'amour que le Platon ficinien est distribué dans les paires Julie/Wolmar, Julie/Saint Preux et Julie/Claire. La spiritualisation de l'amour mis en scène par Rousseau renvoie directement à la radicalisation que Ficin opère dans son commentaire du Banquet entre la Vénus céleste et la Vénus vulgaire ${ }^{74}$. Ce platonisme christianisé est aussi présent chez Malebranche, Lamy, Fénelon. Tous des auteurs que Rousseau a lus ${ }^{75}$. C'est le « platonisme du cœur » dont parle Burgelin. Si le Platon de Ficin repris par Rousseau est un Platon spiritualiste et christianisé, Rousseau découvrira chez Montaigne un Platon moral (accompagné de Sénèque) qu'il va aussi beaucoup utiliser ${ }^{76}$.

Rousseau a ainsi beaucoup fréquenté Platon dans ses lectures. Que ce soit en lisant des traductions de Platon, ou des auteurs citant abondamment Platon, comme Montaigne. On ne peut douter que Platon tienne une place importante dans son «magasin d'idées ». À partir de ce constat, deux gestes herméneutiques peuvent être identifiés. Le premier, dysphorique, prend note de cette importance et s'arrête là. C'est le cas par exemple

72. OC II, p. 223, note.

73. Cf. De Luise \& Farinetti 2001, p. 401-418. Voir aussi Gouhier 1970, p. 153-162.

74. Cf. Marcel 1956, p. 154.

75. Sur Malebranche, cf. Bréhier 1938 et $O C$ I, p. 1111. Sur Fénelon, cf. Riley 2001. Rousseau écrit dans les Confessions qu'il a fait de Lamy son guide. Il dit avoir lu une centaine de fois l'Entretien sur les sciences (OC I, p. 232).

76. Sur Montaigne, $c f$. Vieillard-Baron 1997. Un seul exemple : OC IV, p. 1372 (Rousseau cite les Lois de Platon en passant par Montaigne). 
de Derathé. Le second, euphorique, construit comme comparant un «platonisme » qui est ensuite appliqué à Rousseau. C'est le cas de Williams, qui fait de Rousseau le plus grand platonicien des Lumières, mais aussi de Goldschmidt qui réduit le platonisme à une «structure dualiste » qui informe les principes du système de Rousseau. On peut tenter de se dégager de cette alternative en se centrant sur le platonisme politique. On sait en effet que Rousseau cite majoritairement les dialogues politiques de Platon (Politique, République, Lois). Ce sont aussi ces dialogues qu'il a lus crayon en main dans sa traduction latine de Platon.

\section{Platonisme politique}

Gouhier a noté que Rousseau a gardé de ses lectures de Platon une « tentation platonicienne », que l'on peut illustrer à partir d'un passage du livre VI de la République. Platon y présente l'alternative qui se présente face à l'homme juste confronté à la folie de la multitude: faut-il s'abriter de l'orage derrière un muret, comme un voyageur surpris par la tempête, ou au contraire faire œuvre de législateur et fonder un régime politique juste ${ }^{77}$ ? Gouhier commente :

À certains égards, la vie de Rousseau se joue entre les deux branches de l'alternative posée par Platon : à Venise et jusqu'à la trilogie de la Nouvelle Héloïse, Emile, Le Contrat social, il choisit, comme son maître, le beau risque d'aider les hommes; leur accueil l'obligera à «ne s'occuper que de ses propres affaires », comme disait le Socrate de La République qui fixait alors le programme de vie du Promeneur solitaire. (...) Il y a donc dans la pensée de Rousseau une tentation platonicienne contemporaine de son initiation à la philosophie ${ }^{78}$.

Cette structure a été mise en évidence sous des noms divers : transparence et obstacle (Starobinski), clos et ouvert (Touchefeu ${ }^{79}$. Goldschmidt et Burgelin l'ont souligné, la rhétorique de Rousseau abonde en figures d'opposition et de polarité. L'écriture de Rousseau matérialise ces figures dans un style qui est, selon Burgelin, un piège ${ }^{80}$. Du point de vue qui nous

77. Resp. VI, 496c-497a.

78. Gouhier 1970, p. 141.

79. Cf. Touchefeu 1999, p. 581.

80. Cf. Burgelin 1973, p. 2: «Le texte de Rousseau est un piège : sa méthode d'analyse reste délibérément antinomique. (...) Les notions s'offrent à lui en couples d'opposés : bonté, méchanceté, être, paraître, liberté, esclavage, nature, société. » L'amphibologie pénètre même les concepts de Rousseau: le verbe « dénaturer» a une valeur négative quand il 
intéresse ici, les deux pôles du retrait et de l'engagement public trouvent prima facie une modalisation platonicienne. La tentation platonicienne de Rousseau se réverbère en tentation herméneutique de l'interprète de platoniser Rousseau, faisant l'impasse sur le complexe des médiations. Il nous semble plus prudent de poser que l'écriture de Rousseau recycle un platonisme culturel présent au XVIII ${ }^{e}$ siècle. Le pôle du retrait du solitaire, de la micro-communauté des amants et de la relation pédagogique est construit notamment avec des éléments issus d'un platonisme spiritualiste : c'est le sage solitaire socratique des Promenades, c'est le Platon ficinien dans la Nouvelle Héloïse, qui met en scène un éros spiritualisé ; c'est le Platon de Fénelon dans l'Émile. Le foyer se trouve probablement dans les lectures que Rousseau fit des Oratoriens platonisants. Tournons-nous maintenant vers la question du platonisme politique de Rousseau.

$S$ 'il faut, comme il invite son lecteur à le faire, identifier un principe unifiant la pensée de Rousseau, nous proposons de partir d'un objet paradoxal : il s'agit du grand livre que Rousseau n'a jamais publié : les Institutions politiques. Dans les Confessions, Rousseau écrit que dès 1743-1744, alors qu'il était secrétaire de l'ambassadeur de France à Venise, il eut l'idée d'un grand traité politique :

Des divers ouvrages que j'avais sur le chantier, celui que je méditais depuis longtemps, dont je m'occupais avec le plus de goût, auquel je voulais travailler toute ma vie, et qui devait, selon moi, mettre le sceau à ma réputation, était mes Institutions politiques. [...] J'avais vu que tout tenait radicalement à la politique, et que, de quelque façon qu'on s'y prît, aucun peuple ne serait que ce que la nature de son gouvernement le ferait être ${ }^{81}$.

Rousseau a eu conscience du caractère singulier de ce projet, au point de le garder secret en ne le communiquant pas même à Diderot, qui était alors très proche de lui : « je n'avois voulu communiquer mon projet à personne, pas même à Diderot. Je craignois qu'il ne parut trop hardi pour le siécle et le pays où j'écrivois, et que l'effroi de mes amis ne me gênât dans l'exécution $^{82}$. $\gg$ Ce grand projet semble se présenter, en tout cas rétrospectivement pour Rousseau, comme un foyer d'irradiation, à la fois de grande envergure et secret. Rousseau veut écrire un grand traité de politique. Il n'y parviendra

qualifie l'exercice de la raison; il a une valeur positive quand il qualifie la démiurgie du législateur.

81. OC I, p. 404.

82. OC I, p. 405. 
pas, mais cet échec est en un sens aussi une réussite, puisque c'est à partir de ce projet que Rousseau a écrit de nombreux textes. Dans l'Avertissement du Contrat Social, Rousseau note: «Ce petit traité est extrait d'un ouvrage plus étendu entrepris autrefois sans avoir consulté mes forces, et abandonné depuis longtemps. Des divers morceaux qu'on pouvait tirer de ce qui était fait, celui-ci est le plus considérable $(. . .)^{83} \gg$

Le traité inabouti servira ainsi à la fois de fonds dans lequel Rousseau puisera les matériaux de nombreux livres et de lieu virtuel de convergence ${ }^{84}$. À la fois source et fin, les Institutions politiques sont en quelque sorte un chantier du travail théorique de Rousseau. Les deux Discours, l'article Économie politique pour l'Encyclopédie, l'Émile, les Lettres de la Montagne, les projets de constitution pour la Corse et la Pologne et d'autres fragments politiques et historiques peuvent être vus comme des pièces issues de ce grand projet de traité politique. Tentons de qualifier ce foyer. Il y a d'abord une conception totalisante de la politique. Rousseau est en effet séduit par l'idée d'un primat de la politique, contre une conception sectorielle qui limite la politique à des questions de pouvoir, dans le sillage du paradigme machiavélien. Il chérit l'idée d'une politique architectonique qui associe anthropologie, politique et éducation. Cette thèse peut nous renvoyer à la conception antique de la politique, et plus particulièrement au platonisme politique.

La politique platonicienne construit un projet radical de transformation de l'humain, une politique de l'âme à même de reconfigurer l'anthropologie. Rousseau adopte ce projet «chimérique » platonicien. Mais il semble que les médiations soient là aussi de mise. Le «platonisme politique » de Rousseau passe aussi par des intermédiaires. En l'occurrence, il $s^{\prime}$ agit de sa radicalisation des thèses de Montesquieu ${ }^{85}$. La lecture que Rousseau fait de Platon, et principalement des dialogues politiques, doit être inscrite dans l'histoire de sa réception de L'Esprit des lois.

Commençons par qualifier le geste de Montesquieu. Le premier point à relever est la méthode que Montesquieu utilise, à savoir le comparatisme ${ }^{86}$. Avec L'Esprit des lois, Montesquieu impose un discours comparatiste qui va s'imposer comme méthode d'enquête sur les régimes politiques et les sociétés. La recherche des différences et des ressemblances est déployée aussi

83. OC III, p. 349. Je souligne.

84. Cf. Bernardi 2001, p. 12.

85. Cf. Yack 1986.

86. $C f$. Richter 2006. 
bien sur le terrain historique que géographique. Montesquieu impose cette grammaire comparatiste, entre les pays européens, entre l'Europe et le monde; entre le passé et le présent de l'Europe, principalement l'antiquité gréco-romaine.

Chez Montesquieu, la présence de références à la Grèce antique s’inscrit donc dans un comparatisme. Tout comme pour Rousseau, c'est Plutarque (avec Cicéron) qui est la référence majeure de Montesquieu. Il lui offre un catalogue d'exemples de grands hommes et de grandes institutions. Les conquérants, les philosophes, les mœurs et institutions grecques dressent un portrait à la fois légendaire et exemplaire. Mais les portraits de ces grandeurs anthropologiques et politiques sont contenus dans un cadre sobre qui cherche à mettre en évidence les rapports polyvalents qui constituent l'esprit des lois. Du côté des philosophes, Platon est moins important qu'Aristote pour Montesquieu. Mais dans un cas comme dans l'autre, il lit les textes politiques et laisse de côté la métaphysique et la théorie de la connaissance ${ }^{87}$. Dans le cas de Platon, ce sont évidemment les Lois qui intéressent le juriste Montesquieu. Il en avait copié d'abondants extraits, aujourd'hui perdus mais qu'il mentionne dans ses Pensées. Et L'Esprit des lois mentionne de nombreuses lois présentées dans les $\mathrm{Lois}^{88}$.

L'Esprit des lois paraît en 1748. Rousseau est un de ses premiers lecteurs. En tant que secrétaire de Mme Dupin (depuis 1746), il est chargé de fournir une analyse critique du texte de Montesquieu. La bibliothèque municipale de Bordeaux conserve sept dossiers de notes de lecture de L'Esprit des lois, presque entièrement de la main de Rousseau ${ }^{89}$. Sensible au style et à la pensée du juriste de la Brède, Rousseau va cependant le transformer en radicalisant et en dramatisant Montesquieu. La radicalisation s'appuie sur

87. Montesquieu préfere de loin les Romains, et il goûte peu la philosophie grecque : « La philosophie des Grecs était très peu de chose. Ils ont gâté tout l'univers : non seulement leurs contemporains, mais aussi leurs successeurs. » Montesquieu sauve Platon, parce qu'il est un grand poète: «D'ailleurs, ce n'est plus de la philosophie, mais de la poésie: Platon est un grand poète, de même que le Père Malebranche, Shaftesbury et Montaigne. » (Mes Pensées, OCM I, p. 1545, 1546.)

88. Cf. De l'Esprit des lois, IV, 6: « Ceux qui voudront faire des institutions pareilles établiront la communauté des biens de la république de Platon, ce respect qu'il demandait pour les dieux, cette séparation d'avec les étrangers pour la conservation des mœurs, et la cité faisant le commerce et non pas les citoyens; ils donneront nos arts sans notre luxe, et nos besoins sans nos désirs. » Montesquieu cite plusieurs lois extraites des Lois: lois sur les successions (ibid.V, 5); sur les funérailles (ibid. XXV, 7); sur le suicide (ibid. XXIX, 9).

89. Cf. Ehrard 1997, p. 57. 
un durcissement du comparatisme entre la Grèce antique et l'Europe moderne.

Plus précisément, Rousseau ne se contente pas du comparatisme paratactique de Montesquieu, qui compare des réalités socio-politiques à d'autres réalités. Rousseau axiologise le comparatisme du juriste en comparant les hommes et les réalités antiques et l'Europe du XVIII siècle. Tout comme Montesquieu, Rousseau ne s'intéresse pas à la Grèce antique comme historien. La Grèce n'est pas non plus le berceau de la philosophie. En radicalisant le comparatisme de Montesquieu, Rousseau fait de la Grèce une altérité qui le transporte «dans un autre univers et parmi d'autres êtres $\gg^{90}$. À l'autre bout, la situation des modernes est dramatisée. Si Moïse, Lycurgue et Numa sont de grands législateurs, il n'y a aujourd'hui que des faiseurs de lois. Les hommes de l'histoire ancienne sont de « fortes âmes », aujourd'hui les hommes «se sentent si petits ${ }^{91}$. Cette radicalisation et cette dramatisation du scénario de Montesquieu amènent Rousseau à faire de la Grèce antique un mythe, au centre duquel se trouvent Sparte et ses réalités anthropologiques et socio-juridiques. La radicale altérité des hommes et des institutions antiques fait « qu'ils ne nous ressemblent en rien », comme le dit Rousseau des Lacédémoniens ${ }^{92}$. Rousseau creuse les différences politiques et anthropologiques, là où Montesquieu les posait côte à côte $^{93}$.

Si dans L'Esprit des Lois Montesquieu cherche à réformer les institutions, Rousseau cherchera à utiliser les institutions pour transformer l'homme. La méthode sociologique ante litteram de Montesquieu est un préalable à la position d'une norme radicale, d'une exigence de changer tout le complexe anthropologico-sociologique. Rousseau parle souvent de «chimère » pour qualifier sa démarche. Si Platon est important pour Rousseau, c'est à l'intérieur de ce cadre comparatif qu'il reprend de Montesquieu, qu'il radicalise et complète (la figure de Socrate est absente chez Montesquieu). Si cette hypothèse est correcte, on peut alors dire que les textes politiques

90. OC III, p. 956.

91. Loc. cit. Il y a chez Rousseau un art du contre-pied. Pour lui, les hommes de son temps sont petits, alors précisément que le XVIII ${ }^{c}$ siècle élabore la figure du grand homme et du grand écrivain. $C f$. Bonnet 1998. Rousseau rejette l'histoire moderne et valorise l'histoire ancienne comme magistra vitae.

92. $O C$ III, p. 957.

93. $O C \mathrm{~V}, \mathrm{p} .394$ : «Quand on veut étudier les hommes il faut regarder près de soi ; mais pour étudier l'homme il faut apprendre à porter sa vüe au loin ; il faut d'abord observer les différences pour découvrir les propriétés. » 
de Platon sont des témoins de cette altérité historique, mais qu'ils ne sont pas la source de la politique de Rousseau. Ce sont des témoignages qui livrent des exemples anthropologiques, juridiques et sociologiques. Peu importe alors que ces exemples proviennent des dialogues de Platon ou des Vies de Plutarque. Ce sont l'économie de leur usage, et surtout l'énergie mobilisatrice qu'ils doivent provoquer, qui sont déterminantes.

Pour Rousseau, les institutions politiques ont le pouvoir de briser l'homme - ce qu'elles font ordinairement, et ce qui motive sa critique politique et culturelle. Mais les institutions ont aussi le pouvoir de fabriquer l'homme. Rousseau est conscient de la difficulté de briser le cercle entre anthropologie et politique pour inaugurer un programme de reconfiguration anthropologico-politique. Dans ses Considérations sur le gouvernement de la Pologne et sur sa réformation projettée, il note :

Qu'il soit aisé, si l'on veut, de faire de meilleures loix. Il est impossible d'en faire dont les passions des hommes n'abusent pas, comme ils ont abusé des prémiéres. Prevoir et peser tous ces abus à venir est peut-être une chose impossible à l'homme d'Etat le plus consommé. Mettre la loi au-dessus de l'homme est un problème en politique, que je compare à celui de la quadrature du cercle en géométrie ${ }^{94}$.

Cependant, Rousseau tentera d'affronter la quadrature du cercle politique, sous la guise des deux figures démiurgiques que sont le gouverneur (l'éducateur) et le législateur. Le gouverneur d'Émile s'inscrit dans la figure de Mentor, et le législateur doit être un « grand et puissant génie » ${ }^{95}$. Ces deux figures incarnent la tâche presque impossible de faire des hommes sans les affects micropsychiques et égoïstes de l'amour-propre, sans les fauxsemblants de l'apparaître. Dans l'Émile, Rousseau revient souvent sur l'idée qu'un seul faux pas du gouverneur dans l'éducation de l' « homme rare » ${ }^{96}$, tout est perdu. Cette fragilité est aussi présente dans le Contrat social. La comparaison avec la quadrature du cercle est fort bien choisie : elle pointe à la fois vers une exigence scientifique et vers une impossibilité. Entre le « Newton » de la sociologie que fut Montesquieu et l'utopie politique an-

94. OC III, p. 955. Rousseau l'avait dit aussi dans une lettre à Mirabeau datée du 26 juillet 1767 , qualifiant la question d'une « de ses vieilles idées ». Cf. $O C$ III, p. 1744. Notons en passant que Hobbes pensait avoir résolu la quadrature du cercle politique et celle $\mathrm{du}$ cercle géométrique aussi bien. Sur les aventures de la «résolution» de la quadrature du cercle par Hobbes, voir Bird 1996.

95. OC III, p. 384

96. $O C \mathrm{IV}, \mathrm{p} .251$. 
tique, Rousseau n'a pas voulu choisir. Il semble avoir aggravé ces tensions, au point d'abandonner son projet d'écrire un grand traité de politique.

\section{Conclusion}

La question du platonisme de Rousseau s'inscrit dans un réseau complexe dont nous avons tenté un premier relevé. La multiplicité des platonismes revendiqués par l'historiographie rousseauiste est un premier indice de la difficulté d'isoler un platonisme chez Rousseau. Nous avons vu que Rousseau fait usage dans son écriture de différents platonismes culturels présents au XVIII ${ }^{\mathrm{e}}$ siècle, au premier rang desquels il faut placer le platonisme spiritualiste. Plus remarquable est la configuration d'une nouvelle manière de lire. Rousseau l'a lui-même thématisée : il s'agit d'une lectureévasion qui est aussi une lecture-identification. Les platonismes de Rousseau sont intégrés et réarticulés dans le « complément de subjectivité suggérée ». Mais en même temps, Rousseau accueille les positivités de Montesquieu, qu'il radicalise et dramatise, reconfigurant totalement le régime de réception et de perception des auteurs antiques. Le platonisme politique que Rousseau découvre en lisant les dialogues politiques de Platon devient sous sa plume, à côté d'autres exemples d'institutions et de législateurs antiques, une chimère : une hybridation entre monde moderne et monde antique, entre utopie et réalisme, entre évasion et projet politique, entre philosophie et littérature, entre mythe et concept. D'un point de vue herméneutique aussi, la question du platonisme de Rousseau reste, avec toute son ambivalence, une chimère. 


\section{BIBLIOGRAPHIE}

AYers, M. 2008 : «Introduction », dans: M. Ayers (éd.), Rationalism, Platonism and God: A Symposium on Early Modern Philosophy, Oxford, 2008 (Proceedings of the British Academy, 149), p. 1-14.

Bernardi, B. 2001 : Jean-Jacques Rousseau, Du Contrat social, présentation, notes, bibliographie et chronologie, Paris, 2001 (GF, 1058).

BIRD, A. 1996 : « Squaring the Circle : Hobbes on Philosophy and Geometry », Journal of the History of Ideas, 57 (1996), p. 217-231.

Bonnet, J.-Cl. 1998 : Naissance du Panthéon : essai sur le culte des grands hommes, Paris, 1998 (L'esprit de la cité).

BREHIER, É. 1938 : «Les lectures malebranchistes de Rousseau », Revue internationale de philosophie, 1938, p. 98-120.

Brett A., J. Tully \& H. Hamilton-BleAKLey (éd.) 2006 : Rethinking the Foundations of Modern Political Thought, Cambridge 2006.

Burgelin, P. 1973: La Philosophie de l'existence de Jean-Jacques Rousseau, Paris, ${ }^{2} 1973$ (Bibliothèque d'histoire de la philosophie).

CASSIRER, E. 1932 : «Das Problem Jean Jacques Rousseau », Archiv für Geschichte der Philosophie, 41 (1932), p. 177-213, 479-513. [Réimp. Darmstadt, 1970 (« Libelli », 306) ; trad. fr. par M. B. de Launay, Le problème Jean-Jacques Rousseau, Paris, 1987 (Textes du XX' siècle).]

Compagnon, A. 1979: La Seconde Main ou le travail de la citation, Paris, 1979.

COOPER, L.D. 2002 : « Human Nature and the Love of Wisdom : Rousseau's Hidden (and Modified) Platonism », The Journal of Politics, 64 (2002), p. 108-125.

DE Luise, F. 2000 : «La kallipolis di Rousseau », dans: M. Vegetti (éd.), Platone, La Repubblica. 4, Libro V, Naples, 2000 (Elenchos, 28.4), p. 453-496.

DE LUISE F. \& G. FARINETTI 2001 : Storia della felicitá : gli antichi e i moderni, Turin, 2001 (Piccola biblioteca Einaudi, 110).

Derathe, R. 1955 : «Montesquieu et Jean-Jacques Rousseau », Revue internationale de philosophie, 9 (1955), p. 366-386.

- 1970 : Jean-Jacques Rousseau et la science politique de son temps, Paris, 1970 (Bibliothèque d'histoire de la philosophie).

EHRARD, J. 1997 : «Rousseau et Montesquieu : le mauvais fils réconcilié », Annales de la société Jean-Jacques Rousseau, 41 (1997), p. 57-78.

GAY, P. 1964 : « Reading about Rousseau : a Survey of the Literature » dans : Id., The Party of Humanity: Essays in the French Enlightenment, New York, 1964, p. 211-238.

GolDSCHMIDT, V. 1974 : Anthropologie et politique: les principes du système de Rousseau, Paris, 1974 (Bibliothèque d'histoire de la philosophie).

GOuHIER, H. 1970 : Les Méditations métaphysiques de Jean-Jacques Rousseau, Paris, 1970, $2^{\text {nde }}$ éd. revue 1984 (Bibliothèque d'histoire de la philosophie).

Hampsher-Monk, I., K. Tilmans \& F. Van VRee (éd.) 1998: History of Concepts: Comparative Perspectives, Amsterdam-Londres, 1998.

HeNDEL, Ch.W. 1934 : Jean-Jacques Rousseau Moralist, 2 vol., Londres-New York, 1934.

Hedley, D. \& S. Hutton (éd.) 2008 : Platonism at the Origins of Modernity : Studies on Platonism and Early Modern Philosophy, Dordrecht, 2008 (Archives internationales d'histoire des idées, 196).

HUTTON, S. 2008 : «Introduction », dans : D. Hedley \& S. Hutton 2008, p. 1-8.

JACOB, Ch. (éd.) 2007-2011: Lieux de savoir, 2 vol. [1. Espaces et communautés. 2. Les mains de l'intellect], Paris, 2007, 2011. 
LEIGH, R. A. 1965 : Correspondance complète de Jean-Jacques Rousseau, tome 2 : 1744-1754, édition critique établie et annotée par -, Genève; 1965.

MarCel, R. 1956 : Marsile Ficin, Commentaire sur le Banquet de Platon, texte du manuscrit autographe présenté et traduit par —, Paris, 1956 (Les Classiques de l'Humanisme. Textes, 2).

Nuovo, V. 2008 : « Reflections on Locke's Platonism », dans : D. Hedley \& S. Hutton 2008, p. 207-223.

OC: J.-J. Rousseau, QEuvres complètes, édition publiée sous la direction de B. Gagnebin \& M. Raymond, 5 vol., Paris, 1959, 1961, 1964, 1969, 1995 (Bibliothèque de la Pléiade, 11, 153, 169, 208, 416).

OCM : Montesquieu, CEuvres complètes, texte présenté et annoté par R. Caillois, 2 vol., Paris, 1949, 1951 (Bibliothèque de la Pléiade, 81, 86).

Ottmann, H. H. 2006: Geschichte des politischen Denkens: von den Anfängen bei den Griechen bis auf unsere Zeit. 3.1, Die Neuzeit: von Machiavelli bis zu den grossen Revolutionen, Stuttgart-Weimar, 2006.

RASCHINI, M. A. 2000 : Interpretazioni socratiche, vol. I [seul paru], Milan, 1970 (Pubblicazioni dell'Istituto di Filosofia, Facoltà di Magistero dell'Università di Genova, 10). [2 $2^{\text {nde }}$ éd. a cura di P.P. Ottonello, Venise, 2000 (Scritti di Maria Adelaide Raschini, 5).]

RIBARD, D. 2003 : Raconter, vivre, penser : histoire(s) de philosophes, 1650-1766, Paris, 2003 (Contextes).

RICHEBOURG, M. 1934 : Essai sur les lectures de Rousseau, Genève, 1934.

RiCHTER, M. 2006: «The Comparative Study of Regimes and Societies», dans: M. Goldie \& R. Wokler (éd.), The Cambridge History of Eighteenth-Century Political Thought, Cambridge, 2006, p. 147-171.

RileY, P. 2001 : « Rousseau, Fénelon, and the Quarrel between the Ancients and the Moderns », dans Id. (éd.), The Cambridge Companion to Rousseau, Cambridge, 2001, p. 78-93.

SCHINZ, A. 1934 : [Recension de Hendel 1934], Annales de la Société Jean-Jacques Rousseau, 23 (1934), p. 201-206.

SEUng, T.K. 1996: Plato Rediscovered: Human Value and Social Order, Lanham, Md., 1996 (Studies in Social, Political, and Legal Philosophy).

SEZNEC, J. 1957 : Essais sur Diderot et l'Antiquité, Oxford, 1957.

Silverthorne, M.J. 1973 : «Rousseau's Plato », Studies on Voltaire and the Eighteenth Century, 116 (1973), p. 235-249.

STACKELBERG, J. VON 1958 : « Rousseau, D’Alembert et Diderot traducteurs de Tacite », Studi francesi, 6 (1958), p. 395-407.

STAROBINSKI, J. 1971 : Jean-Jacques Rousseau: la transparence et l'obstacle; suivi de Sept essais sur Rousseau, Paris, 1971 (Bibliothèque des idées). [1 $1^{\text {rc }}$ éd. Paris, 1958.]

TARRANT, H. 2000 : Plato's First Interpreters, Londres-Ithaca, N.Y., 2000.

TOUCHefEU, Y. 1999: L'Antiquité et le christianisme dans la pensée de Jean-Jacques Rousseau, Oxford, 1999 (Studies on Voltaire and the Eighteenth Century, 372).

Trousson, R. 1967 : Socrate devant Voltaire, Diderot et Rousseau : la conscience en face du mythe, Paris, 1967 (Thèmes et mythes, 11).

- 1969: «Diderot helléniste » Diderot Studies, 12 (1969), p. 141-326.

- 1977 : Rousseau et sa fortune littéraire, Paris, 1977 (Tels qu'en eux-mêmes).

VAUGHAN, Ch.E. 1915: The Political Writings of Jean-Jacques Rousseau, edited from the original manuscripts and authentic editions, with introductions and notes by,- 2 vol., Cambridge, 1915. 
Vernes, P.-M. 2009: «Le matérialisme bien tempéré de Rousseau », dans : J. BouladAyoub \& A. Torero-Ibad (éd.), Matérialismes des modernes: nature et moeurs, Québec, 209 (Mercure du Nord), p. 221-238.

Vieillard-Baron, J.-L. 1988 : Platonisme et interprétation de Platon à l'époque moderne, Paris, 1988 (Bibliothèque d'histoire de la philosophie).

- 1997 : «Platonisme éthique et platonisme chrétien: Montaigne, lecteur de Platon », dans : A. B. Neschke-Hentschke (éd.), Images de Platon et lectures de ses auvres : les interprétations de Platon à travers les siècles, Louvain-Paris, 1997 (Bibliothèque philosophique de Louvain, 48), p. 217-236.

VolpilHaC-AUger, C. 1995 : Jean-Jacques Rousseau traducteur de Tacite, présenté et annoté par -, Saint- Etienne, 1995 (Lire le Dix-huitième siècle).

Wallach, J.R. 2001 : The Platonic Political Art : A Study of Critical Reason and Democracy, University Park, Pa., 2001.

Williams, D.L. 2007 : Rousseau's Platonic Enlightenment, University Park, Pa., 2007.

WRIGHT, E.H. 1929 : The Meaning of Rousseau, Londres, 1929.

YACK, B. 1986 : The Longing for Total Revolution: Philosophic Sources of Social Discontent from Rousseau to Marx and Nietzsche, Princeton, 1986 (Studies in Moral, Political and Legal Philosophy). 